\title{
Another Version of Maher's Inequality
}

\author{
Salah Mecheri
}

\begin{abstract}
Let $H$ be a separable infinite dimensional complex Hilbert space, and let $L(H)$ denote the algebra of bounded linear operators on $H$ into itself. Let $A=$ $\left(A_{1}, A_{2} \ldots, A_{n}\right), B=\left(B_{1}, B_{2} \ldots, B_{n}\right)$ be n-tuples of operators in $L(H)$. We define the elementary operator $\Delta_{A, B}: L(H) \mapsto L(H)$ by $\Delta_{A, B}(X)=\sum_{i=1}^{n} A_{i} X B_{i}-X$. In this paper we minimize the map $F_{p}(X)=\left\|T-\Delta_{A, B}(X)\right\|_{p}^{p}$, where $T \in \operatorname{ker} \Delta_{A, B} \cap C_{p}$, and we classify its critical points.
\end{abstract}

Keywords: Orthogonality, derivation, elementary operators

MSC 2000: Primary 47B47, 47A30, 47B20, secondary 47B10

\section{Introduction}

Let $H$ be a separable infinite dimensional complex Hilbert space, and let $L(H)$ denote the algebra of bounded linear operators on $H$ into itself. Given $A, B \in$ $L(H)$, we define the generalized derivation $\delta_{A, B}: L(H) \mapsto L(H)$ by $\delta_{A, B}(X)=$ $A X-X B$. Let $A=\left(A_{1}, A_{2} \ldots, A_{n}\right), B=\left(B_{1}, B_{2} \ldots, B_{n}\right)$ be n-tuples of operators in $L(H)$. We define the elementary operator $\Delta_{A, B}: L(H) \mapsto L(H), \Delta_{A, B}^{*}$ : $L(H) \mapsto L(H)$ by

$$
\Delta_{A, B}(X)=\sum_{i=1}^{n} A_{i} X B_{i}-X
$$

and

$$
\Delta_{A, B}^{*}(X)=\sum_{i=1}^{n} A_{i}^{*} X B_{i}^{*}-X
$$

respectively. Denote $\delta_{A, A}(X)=\delta_{A}(X)=A X-X A$ and $\Delta_{A, A}=\Delta_{A}=$ $\sum_{i=1}^{n} A_{i} X A_{i}-X$. A well-known result of J. Anderson [1: p.136-137] says that if $A$ is a normal operator such that $A S=S A$, then for all $X \in L(H)$,

$$
\|S-(A X-X A)\| \geq\|S\|
$$

Salah Mecheri: Department of Mathematics, King Saud University, College of Science, P.O. Box 2455, Riyadh 11451, Saudi Arabia; mecherisalah@hotmail.com This work was supported by our research center project No. Math/1422/10.

ISSN 0232-2064 / \$ 2.50 (c) Heldermann Verlag Berlin 
The related inequality (1.1) was obtained by P. J. Maher [9: Theorem 3.2]. It shows that, if $A$ is a normal operator and $A S=S A$, where $S \in C_{p}, 1 \leq p<\infty$ and $S \in \operatorname{ker} \delta_{A, B} \cap C_{p}$, then the map $F_{p}$ defined by

$$
F_{p}(X)=\|S-(A X-X A)\|_{p}^{p}
$$

has a global minimizer at $V$ if, and for $1<p<\infty$ only if, $A V-V A=0$. In other words, we have

$$
\|S-(A X-X A)\|_{p} p \geq\|T\|_{p}^{p},
$$

where $C_{p}$ is the von Neumann-Schatten class, $1 \leq p<\infty$ and $\|\cdot\|_{p}$ its norm. In [6] and [3] the authors generalized P. J. Maher's result, showing that if the pair $(A, B)$ has the property $(F P)_{C_{p}}$ (i.e. $A T=T B$, where $T \in C_{p}$ implies $\left.A^{*} T=T B^{*}\right), 1 \leq p<\infty$, and $S \in \operatorname{ker} \delta_{A, B} \cap C_{p}$, then the map $F_{p}$ defined by

$$
F_{p}(X)=\|S-(A X-X B)\|_{p}^{p}
$$

has a global minimizer at $V$ if, and for $1<p<\infty$ only if, $A V-V B=0$. In other words, we have

$$
\|S-(A X-X B)\|_{p}^{p} \geq\|T\|_{p}^{p}
$$

if, and for $1<p<\infty$ only if, $A V-V B=0$. In this paper we obtain an inequality similar to (1.3), where the operator $A X-X B$ is replaced by the operator $\Delta_{A, B}(X)=\sum_{i=1}^{n} A_{i} X B_{i}-X$. We prove that if $\Delta_{A, B}(T)=0=$ $\Delta_{A^{*}, B^{*}}(T)$ and $T \in \operatorname{ker} \Delta_{A, B} \cap C_{p}$, then the map $F_{p}$ defined by

$$
F_{p}(X)=\left\|T-\Delta_{A, B}(X)\right\|_{p}^{p}
$$

has a global minimizer at $V$ if, and for $1<p<\infty$ only if, $\sum_{i=1}^{n} A_{i} V B_{i}-V=0$. Moreover, we show that if $\Delta_{A, B}(T)=0=\Delta_{A^{*}, B^{*}}(T)$ and $T \in \operatorname{ker} \Delta_{A, B} \cap$ $C_{p}, 1<p<\infty$, then the map $F_{p}$ has a critical point at $W$ if and only if $\sum_{i=1}^{n} A_{i} W B_{i}-W=0$, i.e. if $D_{W} F_{p}$ is the Frechet derivative at $W$ of $F_{p}$, then the set

$$
\left\{W \in L(H): D_{W} F_{p}=0\right\}
$$

coincides with ker $\Delta_{A, B}$ (the kernel of $\left.\Delta_{A, B}\right)$.

\section{Preliminaries}

Let $T \in L(H)$ be compact, and let $s_{1}(X) \geq s_{2}(X) \geq \ldots \geq 0$ denote the singular values of $T$, i.e. the eigenvalues of $|T|=\left(T^{*} T\right)^{\frac{1}{2}}$ are arranged in their decreasing order. The operator $T$ is said to belong to the Schatten $p-\operatorname{class} C_{p}$ if

$$
\|T\|_{p}=\left[\sum_{j=1}^{\infty} s_{j}(T)^{p}\right]^{\frac{1}{p}}=\left[\operatorname{tr}(T)^{p}\right]^{\frac{1}{p}}, \quad 1 \leq p<\infty
$$


where $\operatorname{tr}$ denotes the trace function. Hence $C_{1}$ is the trace class, $C_{2}$ is the Hilbert-Schmidt class, and $C_{\infty}$ is the class of compact operators where

$$
\|T\|_{\infty}=s_{1}(T)=\sup _{\|f\|=1}\|T f\|
$$

denotes the usual operator norm. For the general theory of the Schatten $p-$ classes the reader is referred to [11] and [12]. Let $\Re z$ be the real part of a complex number $z, X=U|X|$ be the polar decomposition of the operator $X$ and let $t r$ denote trace.

Theorem 2.1. [2] If $1<p<\infty$, then the map $F_{p}: C_{p} \longmapsto \mathbb{R}^{+}$defined by $X \longmapsto\|X\|_{p}^{p}$, is differentiable at every $X \in C_{p}$ with derivative $\mathcal{D}_{X} F_{p}$ given by

$$
\mathcal{D}_{X} F_{p}(T)=p \cdot \Re \operatorname{tr}\left(|X|^{p-1} U^{*} T\right),
$$

If $\operatorname{dim} \mathrm{H}<\infty$, then the same result holds for $0<p \leq 1$ at every invertible $X$.

Theorem 2.2. [9] If $\mathrm{U}$ is a convex subset of $C_{p}$ with $1<p<\infty$ and $X \in \mathrm{U}$, then the map $X \longmapsto\|X\|_{p}^{p}$ has at most one global minimizer.

Lemma 2.1. [13] Let $C$ denote the n-tuple of operators $\left(C_{1}, C_{2} \ldots, C_{n}\right)$ in $L(H)$. Suppose that $\sum_{i=1}^{n} C_{i} C_{i}^{*} \leq 1$ and $\sum_{i=1}^{n} C_{i}^{*} C_{i} \leq 1$. If $\Delta_{C}(T)=0=\Delta_{C}^{*}(T)$ for some compact operator $T$, then the operator $|T|$ commutes with $C_{i}$ for all $1 \leq i \leq n$.

Definition 2.1. Let $F$ and $G$ be two subspaces of a normed linear space $E$. If $\|x+y\| \geq\|y\|$ for all $x \in F$ and for all $y \in G$, then $F$ is said to be orthogonal to $G$.

\section{Main Results}

Let $\mathcal{U}(A, B)=\left\{X \in L(H):\left(\sum_{i=1}^{n} C_{i} X C_{i}-X\right) \in C_{p}\right\}$ and $F_{p}: \mathcal{U} \longmapsto \mathbb{R}^{+}$be the map defined by $F_{p}(X)=\left\|T-\left(\sum_{i=1}^{n} C_{i} X C_{i}-X\right)\right\|_{p}^{p}$, where $T \in \operatorname{ker} \Delta_{C} \cap$ $C_{p}, 1 \leq p<\infty$. We start with the following lemma which will be used in the proof of Theorem 3.1 .

Lemma 3.1. Let $C$ denote the n-tuple of operators $\left(C_{1}, C_{2} \ldots, C_{n}\right)$ in $L(H)$ such that $\sum_{i=1}^{n} C_{i} C_{i}^{*} \leq 1, \sum_{i=1}^{n} C_{i}^{*} C_{i} \leq 1$. Let $S$ be compact and $\Delta_{c}(S)=0=$ $\Delta_{c}^{*}(S)$. If

$$
\sum_{i=1}^{n} C_{i}|S|^{p-1} U^{*} C_{i}=|S|^{p-1} U^{*},
$$

where $p>1$ and $S=U|S|$ is the polar decomposition of $S$, then

$$
\sum_{i=1}^{n} C_{i}|S| U^{*} C_{i}=|S| U^{*} .
$$


Proof. If $T=|S|^{p-1}$, then

$$
\sum_{i=1}^{n} C_{i} T U^{*} C_{i}=T U^{*}
$$

We prove that

$$
\sum_{i=1}^{n} C_{i} T^{n} U^{*} C_{i}=T^{n} U^{*} .
$$

It is known that if $\sum_{i=1}^{n} C_{i} C_{i}^{*} \leq 1, \sum_{i=1}^{n} C_{i}^{*} C_{i} \leq 1$ and $\Delta_{c}(S)=0=\Delta_{c}^{*}(S)$, then the eigenspaces corresponding to distinct non-zero eigenvalues of the compact positive operator $|S|^{2}$ reduce each $C_{i}$ (see [4: Theorem 8], [13: Lemma 2.3]). In particular, $|S|$ commutes with $C_{i}$ for all $1 \leq i \leq n$. This implies also that $|S|^{p-1}=T$ commutes with each $C_{i}$ for all $1 \leq i \leq n$. Hence

$$
C_{i}|T|=|T| C i
$$

and $C_{i} T^{2}=T^{2} C_{i}$. Since $C_{i}$ commutes with the positive operator $T^{2}$, then $C_{i}$ commutes with its square root, that is

$$
C_{i} T=T C_{i}
$$

By (3.3) and (3.1), we obtain(3.2).

By using an argument similar to the proof of Theorem 3.2 in [9], we can consider the map $f$ defined on $\sigma(T) \subset \mathbb{R}^{+}$by $f(t)=t^{\frac{1}{p-1}}, 1<p<\infty$. Since $f$ is the uniform limit of a sequence $\left(P_{i}\right)$ of polynomials without constant term (since $f(0)=0)$, it follows from (3.2) that $\sum_{i=1}^{n} C_{i} P_{i}(T) U^{*} C_{i}=P_{i}(T) U^{*}$. Therefore $\sum_{i=1}^{n} C_{i} T^{\frac{1}{p-1}} U^{*} C_{i}=U^{*} T^{\frac{1}{p-1}}$.

Now we are ready to present our first result on the global minimizer.

Theorem 3.1. Let $C=\left(C_{1}, C_{2} \ldots, C_{n}\right)$ be an n-tuple of operators in $L(H)$. If

$$
\begin{gathered}
\sum_{i=1}^{n} C_{i} C_{i}^{*} \leq 1, \quad \sum_{i=1}^{n} C_{i}^{*} C_{i} \leq 1, \\
\Delta_{c}(T)=0=\Delta_{c}^{*}(T)
\end{gathered}
$$

and $T \in \operatorname{ker} \Delta_{A, B} \cap C_{p}$, then for $1 \leq p<\infty$, the map $F_{p}$ has a global minimizer at $W \in L(H)$ if, and for $1<p<\infty$ only if,

$$
\sum_{i=1}^{n} C_{i} W C_{i}-W=0
$$


Proof. If

$$
\sum_{i=1}^{n} C_{i} W C_{i}-W=0
$$

then $F_{p}(W)=\|T\|_{p}^{p}$. It follows from [13: Theorem 2.4] that

$$
F_{p}(X) \geq F_{p}(W) .
$$

Conversely, if $F_{p}$ has a minimum then

$$
\left\|T-\left(\sum_{i=1}^{n} C_{i} W C_{i}-W\right)\right\|_{p}^{p}=\|T\|_{p}^{p} .
$$

Since $\mathcal{U}$ is convex, the set $\mathcal{V}=\left\{T-\left(\sum_{i=1}^{n} C_{i} X C_{i}-X\right) ; X \in \mathcal{U}\right\}$ is also convex. Thus, Theorem 2.2 implies that $T-\left(\sum_{i=1}^{n} C_{i} W C_{i}-W\right)=T$.

In the following theorem we will classify the critical points of the map $F_{p}(p>1)$.

Theorem 3.2. Let $C=\left(C_{1}, C_{2} \ldots, C_{n}\right)$ be an $n$-tuple of operators in $L(H)$. If

$$
\begin{gathered}
\sum_{i=1}^{n} C_{i} C_{i}^{*} \leq 1, \sum_{i=1}^{n} C_{i}^{*} C_{i} \leq 1, \\
\Delta_{c}(T)=0=\Delta_{c}^{*}(T)
\end{gathered}
$$

and $T \in \operatorname{ker} \Delta_{A, B} \cap C_{p}$, then for $1 \leq p<\infty$, the map $F_{p}$ has a critical point at $W \in L(H)$ if, and for $1<p<\infty$ only if,

$$
\sum_{i=1}^{n} C_{i} W C_{i}-W=0
$$

Proof. Since the Frechet derivative of $F_{p}$ is given by

$$
\mathcal{D}_{W} F_{p}(T)=\lim _{h \longrightarrow 0} \frac{F_{p}(W+h T)-F_{p}(W)}{h},
$$

it follows that

$$
\mathcal{D}_{W} F_{p}(T)=\left[\mathcal{D}_{S-\left(\sum_{i=1}^{n} C_{i} W C_{i}-W\right)}\right]\left(\sum_{i=1}^{n} C_{i} T C_{i}-T\right) .
$$

If $W$ is a critical point of $F_{p}$, then $\mathcal{D}_{W} F_{p}(T)=0 \forall T \in \mathcal{U}$. By applying Theorem 2.1 we get

$$
\begin{aligned}
\mathcal{D}_{W} F_{p}(T) & =p \Re \operatorname{tr}\left[\left|S-\left(\sum_{i=1}^{n} C_{i} W C_{i}-W\right)\right|^{p-1} U_{1}^{*}\left(\sum_{i=1}^{n} C_{i} T C_{i}-T\right)\right] \\
& =p \Re \operatorname{tr}\left[Y\left(\sum_{i=1}^{n} C_{i} T C_{i}-T\right)\right] \\
& =0
\end{aligned}
$$


where $S-\left(\sum_{i=1}^{n} C_{i} W C_{i}-W\right)=U_{1}\left|S-\left(\sum_{i=1}^{n} C_{i} W C_{i}-W\right)\right|$ is the polar decomposition of the operator $S-\left(\sum_{i=1}^{n} C_{i} W C_{i}-W\right)$ and

$$
Y=\left|S-\left(\sum_{i=1}^{n} C_{i} W C_{i}-W\right)\right|^{p-1} U_{1}^{*}
$$

An easy calculation shows that $\left(\sum_{i=1}^{n} C_{i} Y C_{i}-Y\right)=0$, that is

$$
\sum_{i=1}^{n} C_{i}\left|S-\left(\sum_{i=1}^{n} C_{i} W C_{i}-W\right)\right|^{p-1} U_{1}^{*} C_{i}=\left|S-\left(\sum_{i=1}^{n} C_{i} W C_{i}-W\right)\right|^{p-1} U_{1}^{*}
$$

It follows from Lemma 3.1 that

$$
\sum_{i=1}^{n} C_{i}\left|S-\left(\sum_{i=1}^{n} C_{i} W C_{i}-W\right)\right| U_{1}^{*} C_{i}=\left|S-\left(\sum_{i=1}^{n} C_{i} W C_{i}-W\right)\right| U_{1}^{*} .
$$

By taking adjoints and since $\Delta_{C}=0=\Delta_{C^{*}}$, we get

$$
\sum_{i=1}^{n} C_{i}\left(T-\left(\sum_{i=1}^{n} C_{i} W C_{i}-W\right)\right) C_{i}=\left(T-\left(\sum_{i=1}^{n} C_{i} W C_{i}-W\right)\right) .
$$

Then

$$
\sum_{i=1}^{n} C_{i}\left[\left(\sum_{i=1}^{n} C_{i} W C_{i}-W\right)\right] C_{i}=\left(\sum_{i=1}^{n} C_{i} W C_{i}-W\right)
$$

Hence

$$
\sum_{i=1}^{n} C_{i} W C_{i}-W \in R\left(\Delta_{C}\right) \cap \operatorname{ker} \Delta_{C},
$$

where $R\left(\Delta_{C}\right)$ is the range of $\Delta_{C}$. It is easy to see that (arguing as in the proof of [13: Theorem 2.4]), $\Delta_{C}(T)=0=\Delta_{C^{*}}(T)$ and $T \in \operatorname{ker} \Delta_{C}$, where $T \in L(H)$. Then

$$
\| T-\Delta_{C}(X\|\geq\| T \|
$$

holds for all $X \in L(H)$ and for all $T \in \operatorname{ker} \Delta_{c}$. Hence $\sum_{i=1}^{n} C_{i} W C_{i}-W=0$. Conversely, if $\sum_{i=1}^{n} C_{i} W C_{i}=W$, then $W$ is a minimum of $F_{p}$, and since $F_{p}$ is differentiable, $W$ is a critical point.

In the above theorem we classified the critical points of the map $F_{p}$ for $p>1$. In the following theorem we consider the case $0<p \leq 1$.

Theorem 3.3. Let $C=\left(C_{1}, C_{2} \ldots, C_{n}\right)$ be an n-tuple of operators in $L(H)$. If

$$
\sum_{i=1}^{n} C_{i} C_{i}^{*} \leq 1, \quad \sum_{i=1}^{n} C_{i}^{*} C_{i} \leq 1
$$

such that $\Delta_{C}(S)=0=\Delta_{C^{*}}(S)$ and $S \in \operatorname{ker} \Delta_{C} \cap C_{p}, 0<p \leq 1, \operatorname{dim} H<\infty$ and $S-\left(\sum_{i=1}^{n} C_{i} W C_{i}-W\right)$ is invertible, then $F_{p}$ has a critical point at $W$, if $\sum_{i=1}^{n} C_{i} W C_{i}-W=0$. 
Proof. Let $W, S \in U$ and let $\phi$, be the map defined by

$$
\phi: X \longmapsto S-\left(\sum_{i=1}^{n} C_{i} X C_{i}-X\right) .
$$

Suppose that $\operatorname{dim} H<\infty$. If $\sum_{i=1}^{n} C_{i} W C_{i}-W=0$, then $S$ is invertible by hypothesis. Also $|S|$ is invertible, hence $|S|^{p-1}$ exists for $0<p \leq 1$. Taking $Y=|S|^{p-1} U^{*}$, where $S=U|S|$ is the polar decomposition of $S$. As shown in Lemma 3.1, $|S|$ commutes with $C_{i}$ for all $1 \leq i \leq n$. Hence

$$
C_{i}|S|=|S| C_{i}
$$

Since $\sum_{i=1}^{n} C_{i} S^{*} C_{i}=S^{*}$, i.e.

$$
\sum_{i=1}^{n} C_{i}|S| U^{*} C_{i}=|S| U^{*},
$$

we find

$$
|S|\left(\sum_{i=1}^{n} C_{i} U^{*} C_{i}-U^{*}\right)=0,
$$

and since

$$
A|S|^{p-1}=|S|^{p-1} A,
$$

we have

$$
\begin{aligned}
\sum_{i=1}^{n} C_{i} Y C_{i}-Y & =\sum_{i=1}^{n} C_{i}|S|^{p-1} U^{*} C_{i}-|S|^{p-1} U^{*} \\
& =|S|^{p-1}\left(\sum_{i=1}^{n} C_{i} U^{*} C_{i}-U^{*}\right),
\end{aligned}
$$

so that $\sum_{i=1}^{n} C_{i} Y C_{i}-Y=0$ and $\operatorname{tr}\left[\left(\sum_{i=1}^{n} C_{i} Y C_{i}-Y\right) T\right]=0$ for all $T \in L(H)$. Since

$$
S=S-\left(\sum_{i=1}^{n} C_{i} W C_{i}-W\right),
$$

we have

$$
\begin{aligned}
0 & =\operatorname{tr}\left[Y\left(\sum_{i=1}^{n} C_{i} T C_{i}-T\right)\right] \\
& =p \Re \operatorname{tr}\left[Y\left(\sum_{i=1}^{n} C_{i} T C_{i}-T\right)\right] \\
& =p \Re \operatorname{tr}\left[|S|^{p-1} U^{*}\left(\sum_{i=1}^{n} C_{i} T C_{i}-T\right)\right] \\
& =\left(\mathcal{D}_{S} \phi\right)\left(\sum_{i=1}^{n} C_{i} T C_{i}-T\right)
\end{aligned}
$$




$$
=\left(\mathcal{D}_{W} F_{p}\right)(T)
$$

which proofs the assertion.

At the end we use a familar device of considering $2 \times 2$ operator matrices to extend the previous theorems to the elementary operator $\sum_{i=1}^{n} A_{i} X B_{i}-X$.

Theorem 3.4. Let $A=\left(A_{1}, A_{2} \ldots, A_{n}\right), B=\left(B_{1}, B_{2} \ldots, B_{n}\right)$ be $n$-tuples of operators in $L(H)$ such that

$$
\sum_{i=1}^{n} A_{i} A_{i}^{*} \leq 1, \sum_{i=1}^{n} A_{i}^{*} A_{i} \leq 1, \sum_{i=1}^{n} B_{i} B_{i}^{*} \leq 1, \sum_{i=1}^{n} B_{i}^{*} B_{i} \leq 1 .
$$

If $\Delta_{A, B}(T)=0=\Delta_{A, B}^{*}(T)$ and $T \in \operatorname{ker} \Delta_{A, B} \cap C_{p}$, then it holds for $1 \leq p<\infty$ :

(i) the map $F_{p}$ has a global minimizer at $W$ if, and for $1<p<\infty$ only if, $\sum_{i=1}^{n} A_{i} W B_{i}-W=0$

(ii) the map $F_{p}$ has a critical point at $W$ if, and for $1<p<\infty$ only if, $\sum_{i=1}^{n} A_{i} W B_{i}-W=0$

(iii) the map $F_{p}, 0<p \leq 1$, has a critical point at $W$ if $\sum_{i=1}^{n} A_{i} W B_{i}-W=0$ provided $\operatorname{dim} H<\infty$ and $S-\left(\sum_{i=1}^{n} A_{i} W B_{i}-W\right)$ is invertible.

Proof. It suffices to take the Hilbert space $H \oplus H$, and operators

$$
C_{i}=\left[\begin{array}{ll}
A_{i} & 0 \\
0 & B_{i}
\end{array}\right] S=\left[\begin{array}{ll}
0 & T \\
0 & 0
\end{array}\right], X=\left[\begin{array}{ll}
0 & X \\
0 & 0
\end{array}\right]
$$

and apply Theorem 3.1, Theorem 3.2 and Theorem 3.3. These arguments use operator matrices as in Bouali and Cherki [3] and Mecheri [7].

\section{Remark.}

1. In Theorem 3.2, the implication

$$
W \text { is a critical point } \Longrightarrow \sum_{i=1}^{n} A_{i} W B_{i}-W=0
$$

does not hold in the case $0<p \leq 1$ (cf. Maher [8]).

2. Theorems 3.1, 3.2, 3.3 and 3.4 hold in particular if $A$ and $B$ are contractions. Indeed, it is known from [4] that if $A$ and $B$ are contractions and $\Delta_{A, B}(S)=A S B-S=0$, where $S \in C_{p}$, then

$$
\Delta_{A^{*}, B^{*}}(S)=\delta_{A^{*}, B}(S)=\delta_{A, B^{*}}(S)=0 .
$$

3. If $A \in C_{p}$, the conclusions of Theorems 3.1, 3.2, 3.3 and 3.4 hold for all $X \in L(H)$ (cf. Maher [9]). 
Acknowledgment. The author would like to thank the referee for his careful reading of the paper. His valuable suggestions, critical remarks, and pertinent comments made numerous improvements throughout.

\section{References}

[1] Anderson, J. H.: On normal derivations. Proc. Amer. Math. Soc. 38 (1973), $135-140$.

[2] Almoadjil, A. H.: The commutants of relatively prime powers in Banach algebra. Proc. Amer. Math. Soc. 57 (1976), 243 - 251.

[3] Bouali, S. and S. Cherki: Approximation by generalized commutators. Acta Sci. Math. (Sczeged) 63 (1991), $213-218$.

[4] Douglas, R.G.: On the operator $S^{*} X T=X$ and related topics. Acta sci. Math. (Szeged) 30 (1969), 19 - 32.

[5] Duggal, B. P.: On intertwining operators. Monatsh. Math. 106 (1988), 139 148.

[6] Gohberg, I. C. and M. G. Krein: Introduction to the Range of Linear Nonselfadjoint Operators. Trans. Math. Monogr. 18. Providence: Amer. Math. Soc. 1969.

[7] Mecheri, S.: On minimizing $\| S-\left(A X-X B \|_{p}^{p}\right.$. Serdica. Math. J. 26 (2000), $119-126$.

[8] Mecheri, S.: Non-normal derivation and orthogonality. Proc. Amer. Math. Soc. (in press).

[9] Maher, P. J.: Commutator approximants. Proc. Amer. Math. Soc. 115 (1992), $995-1000$.

[10] Maher, P. J.: Some norm inequalities concerning generalized inverses. Lin. Alg. Appl. 174 (1992), 951 - 960.

[11] Schatten, R.: Norm Ideals of Completely Continuous Operators. Berlin: Springer-Verlag 1960.

[12] Simon, B.: Trace Ideals and Their Applications. Cambridge: Cambridge Univ. Press 1979.

[13] Turns̃ek, A.: Elementary operators and orthogonality. Lin. Alg. Appl. 317 (2000), $207-216$

Received 10.10.2002; in revised form 13.01.2004 